\title{
ESTUDIO PARA EL DISEÑO Y EVALUACIÓN DE UN SISTEMA DESALINIZADOR HÍBRIDO EN TUMACO
}

\section{Environmental Design and Evaluation of a Hybrid Watermaker}

\author{
LEONARDO JARAMILLO ${ }^{1}$ \\ ${ }_{1}$ Universidad del Valle
}

Emotivo homenaje póstumo

Recibido: 26 de Junio de 2010

Aceptado: 11 de Enero de 2011

\section{Resumen}

El agua es un recurso imprescindible para el desarrollo de la vida. Se estima que solo el 0,03 \% del volumen total del planeta es agua dulce disponible para el hombre, aunque también inicialmente es inaccesible. Adicionalmente la contaminación, el mal uso, los costos de captación, transporte y potabilización lo convierten en un recurso limitado que debe preservarse. Este artículo propone evaluar y diseñar un modelo de desalinización en zona costera de Tumaco utilizando sistemas híbridos y sensores bioinspirados bajo una racionalidad ambiental del agua indispensable para la comunidad incorporando tecnologías de producción y consumo sostenible.

Palabras claves: PML, Análisis Multicriterio Social, Desalinización, Fuentes Renovables de Energía, Sistemas híbridos, Racionalidad ambiental, Bioinspiración, Complementariedad, Comunidad Impactos ambientales

\begin{abstract}
.
The water is an essential resource for development of life. According to estimates, only $0.03 \%$ of the total earth's volume is fresh water, available for mankind, although is also inaccessible. Additionally, contamination, wrong use of this resource and costs of water harvesting, transport and purification, become fresh water in a quite limited resource that must be preserved. This paper proposes to evaluate and design a watermaker model in a Tumaco coast zone using hybrid systems and bioinspired sensors depending on a environmental rationality, allowing the use of fresh water for community, involving sustainable production and consumption technologies.
\end{abstract}

Keywords: PML, Social multi-criteria analysis, Watermarker, Renewable Energy Sources, Hybrid systems, Rural environmental management, Bioinspiración, Complementarity, Community, Environmental impacts. 


\section{INTRODUCCIÓN}

La escasez de agua dulce es uno de los siete problemas ambientales fundamentales presentados en el Informe "Perspectivas del Medio Ambiente Mundial" del PNUMA. Se está alcanzando el límite de extraer agua dulce de la superficie terrestre, pero el consumo no deja de aumentar. Sin embargo, una gran amenaza la constituye el efecto que el cambio climático tendrá sobre el ciclo hidrológico y la disponibilidad de agua dulce donde se agravarán las condiciones de escasez de las zonas que ya son áridas (menos lluvias y mayor evaporación).

En todo el mundo la población está concentrada a lo largo o cerca de la costa, ocupando sólo el $10 \%$ de la zona continental de la tierra. En las zonas costeras, las actividades humanas están en vías de aniquilar los ecosistemas costeros y oceánicos y la riqueza de la biodiversidad que éstos albergan.

Las zona costera de Tumaco como caso de estudio contiene: crecimiento demográfico, producción de pobreza siendo zonas con alta biodiversidad y no cuentan con agua dulce en sitio, por lo cual es pertinente la búsqueda de soluciones interdisciplinarias en la complementariedad entre calidad de vida-producción más limpia, que involucren la participación pública de la comunidad, gobierno, acompañamiento institucional, desarrollos de tecnología con la comunidad y sostenibilidad.

Debido a que hay dificultad en la consecución del agua dulce en la zona costera de Tumaco y dada la abundancia del agua marina (agua salada y salobre) se plantea la obtención del agua dulce en dicha zona mediante el diseño y evaluación ambiental de un modelo desalinizador híbrido utilizando sensores bioinspirados.

El desarrollo de este proyecto se plantea bajo el mejoramiento de la calidad de vida en la comunidad, alimentación, prevención de las enfermedades, disminución de la pobreza y problemas sociales.

\section{DESCRIPCIÓN}

El agua es un recurso natural no renovable que ha alcanzado niveles alarmantes de escasez. Este un recurso imprescindible para el desarrollo de la vida. Se estima que solo el $0,03 \%$ del volumen total del planeta es agua dulce disponible para el hombre, aunque también inicialmente es inaccesible. Ya que está, en su mayoría, como se dijo anteriormente se encuentra en las zonas polares y en los glaciares o en acuíferos profundos poco accesibles, lo que deja tan solo un $0,003 \%$ del volumen total de agua sobre la corteza terrestre directamente accesible para el ser humano. Adicionalmente la contaminación, el mal uso, los costos de captación, transporte y potabilización lo convierten en un recurso limitado que debe preservarse.

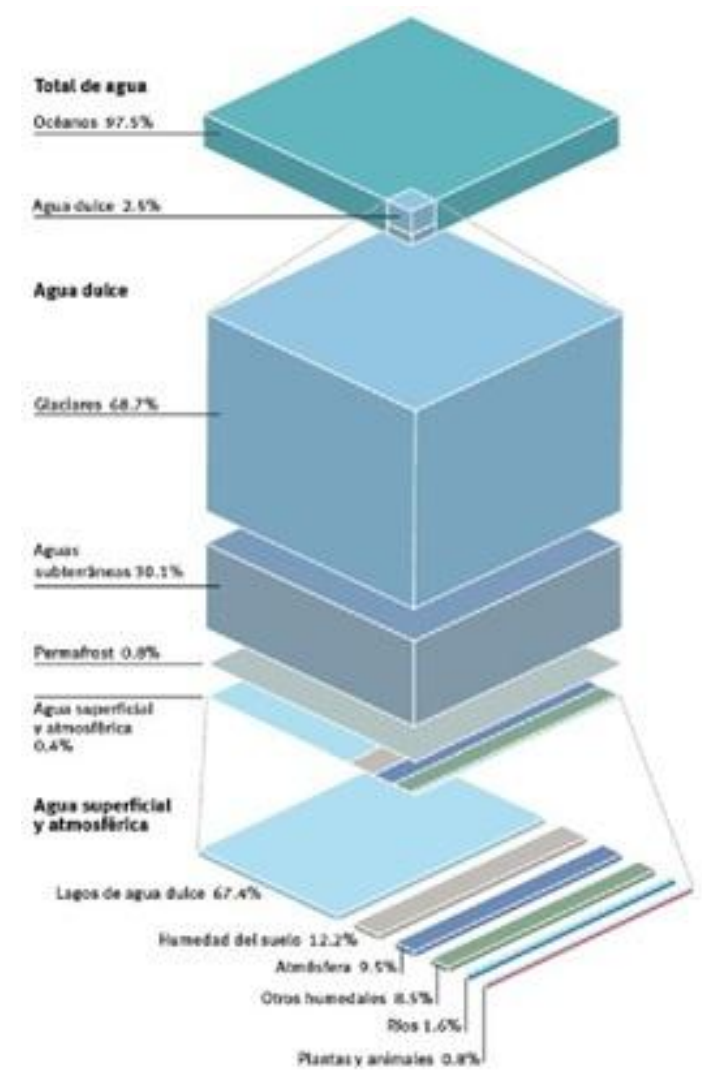

Figura 1. Distribución del agua en el mundo, Tomado de Programa de Naciones Unidas (PNUMA).

La creciente necesidad de lograr el equilibrio hidrológico que asegure el abasto suficiente de agua a la población se puede lograr armonizando la disponibilidad natural con las extracciones del recurso mediante el uso eficiente del agua.

La tendencia del uso de este recurso en Colombia en los últimos años, ha evidenciado que el $70 \%$ del agua que se utiliza corresponde a riego agrícola, lo cual denota la importancia que tiene este bien para la comunidad. La industria y la minería no consumen más que el promedio mundial, mientras que el agua para uso domestico en nuestro país es cercano al $4 \%$ de la disponible, lo cual es la mitad del promedio mundial. 


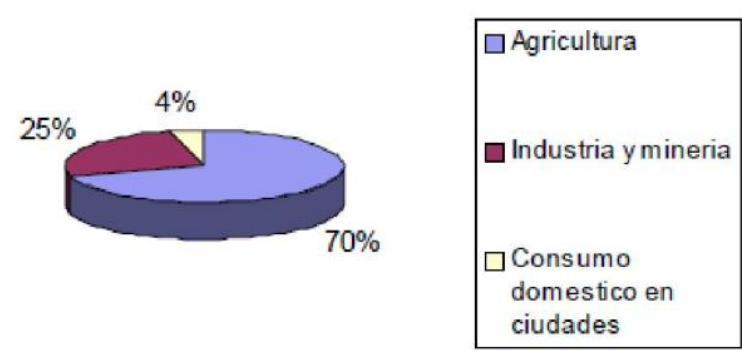

Figura 2. Usos del Agua en Colombia

Surge a continuación esta pregunta: ¿Por qué teniendo un gran porcentaje de agua del mar en una zona costera no se cuenta con agua dulce?

No es nueva la problemática que ocurre en el mundo sobre abastecimiento de agua dulce para las comunidades costeras, de hecho la UNESCO predice que para el año o 2020 la escasez de agua se convertirá en uno de los peores problemas alrededor del mundo.

La escasez de agua dulce es uno de los siete problemas ambientales fundamentales presentados en el Informe "Perspectivas del Medio Ambiente Mundial" del PNUMA. Se está alcanzando el límite de extraer agua dulce de la superficie terrestre, pero el consumo no deja de aumentar. Sin embargo, una gran amenaza la constituye el efecto que el cambio climático tendrá sobre el ciclo hidrológico y la disponibilidad de agua dulce donde se agravarán las condiciones de escasez de las zonas que ya son áridas (menos lluvias y mayor evaporación).

En todo el mundo la población está concentrada a lo largo o cerca de la costa, ocupando sólo el $10 \%$ de la zona continental de la tierra. En las zonas costeras, las actividades humanas están en vías de aniquilar los ecosistemas costeros y oceánicos y la riqueza de la biodiversidad que éstos albergan.

Con base en los Patrones demográficos, en todo el mundo los habitantes se aglutinan cerca de la costa. Más de la mitad de la población mundial - unos 3.200 millones - ocupan una zona costera de 200 kilómetros de ancho. En Colombia, Tumaco concentra el 65 por ciento de los habitantes de la subregión Pacífica. Los negros y mulatos representan el 93 $\%$ de la población de la subregión, los indígenas el 6 \% y los blancos y mestizos el $1 \%$.

Tumaco se ha caracterizado por el abandono estatal manifestado en los más bajos índices de cobertura de la población en cuanto a servicios de educación, salud, vivienda, acueducto y alcantarillado, comunicaciones, entre otros y por el establecimiento de economías de enclave que han dejado a sus pobladores en mayor pobreza. Las comunidades han resistido a dicho abandono, gracias a su capacidad adaptativa, a su creatividad para generar alternativas de desarrollo y manejo del medio ambiente, a su capacidad autogestionaria para sobrevivir en medio de condiciones adversas.

El clima de Tumaco se enmarca dentro de las características generales de la zona de convergencia intertropical como son altas temperaturas aunque no excesivas, aire húmedo $\mathrm{y}$ abundantes lluvias. Influye de manera fundamental el relieve de Tumaco que es plano y a nivel del mar con elevaciones que no superan los 50 metros de altura; adicionalmente, condiciona las características climáticas el frente montañoso andino que limita al oriente la Llanura Costera del Pacífico y actúa como una barrera que detiene el flujo, del mar hacia el continente, de los vientos cargados de humedad y los obliga a ascender.

La economía de la región costera del Pacífico nariñense se basa principalmente en la agricultura (agroindustria), la pesca, la actividad forestal y el turismo: en Tumaco se produce el $100 \%$ de la palma africana, el $92 \%$ del cacao y el $51 \%$ del coco de Nariño, y también se concentra gran parte de la oferta hotelera departamental. Tumaco es también el principal puerto petrolero colombiano sobre el océano Pacífico y el segundo a nivel nacional, después de Coveñas.

La parte Geomorfológica y geológica de Tumaco como se describe en el informe de INGEOMINAS (Nivia et al. 2003) se han desarrollado deltas (estructuras convexas que destacan en la costa en la desembocadura de un rio) sobre un sustrato constituido por rocas sedimentarias neógenas que aflora en la costa acantilada localizada al interior de la Bahía, que sirve de separación a los dos sistemas de deltas. El sustrato rocoso está constituido por la Formación Mayorquín que aflora en pequeñas colinas que no superan los $100 \mathrm{~m}$ de altura, por esto, aunque las rocas son blandas, y susceptibles a los fenómenos de erosión física y química, los fenómenos de remoción en masa no tienen mayor importancia, excepto en los acantilados donde ocurre, pérdida de soporte en la base de los taludes por erosión litoral.

Las zona costera de Tumaco como caso de estudio contiene: crecimiento demográfico, producción de pobreza siendo zonas con alta biodiversidad y no cuentan con agua dulce en sitio debido a que deben desplazarse entre 2 o tres horas diarias para traer el líquido necesario y con difícil acceso de carreteras como lo menciona (Zúñiga 1993), por lo cual es pertinente la búsqueda de soluciones interdisciplinarias en la complementariedad entre calidad de vida-Producción más limpia, que involucren la participación pública de la 
comunidad, gobierno, acompañamiento institucional, desarrollos de tecnología con la comunidad y sostenibilidad.

El presente estudio tiene el objetivo de diseñar y evaluar ambientalmente un sistema desalinizador híbrido utilizando sensores bioinspirados en la zona costera del municipio de Tumaco. Por medio de la exploración, identificación y análisis de las distintas variables que implica el manejo y uso sustentable y eficiente del recurso bajo una racionalidad ambiental y comprendiendo las diversas áreas de influencia del mar, de la comunidad y sintetizar los hallazgos en indicadores que permitan comparar si hay un avance o un retroceso hacia la comunidad e impactos ambientales.

Para ello se utiliza la Evaluación Multicriterio Social (Munda 2002), metodología que selecciona y posiciona alternativas de acuerdo a criterios cualitativos, cuantitativos o ambos a la vez. Posibles alternativas serán tecnologías sustentables en la región, reducción de impactos ambientales bajo una producción más limpia, y posibles criterios de evaluación serán dimensiones y categorías que se identifican a través de una investigación primaria de entrevistas a profundidad (acción participativa) y de un estudio del estado del arte. El resultado esperado no se basa solamente en saber qué diseño y su evaluación ambiental respectiva, sino en un análisis desagregado para identificar la evolución por separado de cada componente tecnológico, ambiental, cultural y territorial incluido en el estudio, dentro de un marco conceptual definido.

\section{Clasificación de Procesos de desalinización}

Aunque existen diversos criterios para clasificar los diferentes procesos de desalinización, un modo útil y claro de clasificarlos es dividirlos en aquellos que utilizan energía no renovable y los que usan energía renovable. Los procesos que utilizan energía no renovable comprenden dos grupos, aquellos que implican un cambio de fase en el agua y los que funcionan sin cambio de fase. Los métodos con cambio de fase más utilizados son Destilación en Múltiple Efecto (MED) y Flashing en Múltiple Efecto (MEF) y los que funcionan sin cambio de fases son Osmosis Inversa (OI) y Electrodiálisis (Zarza 2005). Los procesos que utilizan energías renovables se basan en el uso de las experiencias llevadas a cabo sobre Plataformas Solares como son Solar Still, Colectores Desalinizadores Compactos (CDC), Sistemas Solares de Desalinización con Plantas MED o MEF, Plantas Desalinizadoras Fotovoltaicas y Eólicas (Urrutia 2001).

\section{Aspectos energéticos de la desalinización}

Los costos de producción de agua dulce en el proceso de desalinización se muestran en la figura 2. El costo debido al consumo de energía, por parte del proceso es superior al $40 \%$ del costo total del agua desalinizada.

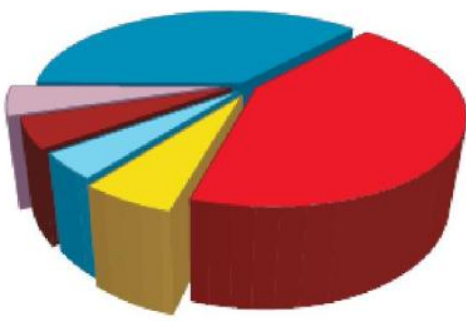

$$
\begin{array}{r|ll|l}
\text { 43\% } & \text { Energia } & 5 \% & \text { Sustitución de membranas } \\
37 \% & \text { Amortización } & 5 \% & \text { Mano de obra } \\
\hline 6 \% & \text { Mantenimiento; otros } & 4 \% & \text { Productos químicos; consumibles }
\end{array}
$$

Figura 2. Porcentajes de Participación en el costo del agua para desalinización, tomado de Fariñas (2008).

\section{Parámetros que intervienen en el consumo específico de energía}

El consumo específico de energía en la desalinización del agua de mar depende de varios parámetros como:

- $\quad$ La temperatura del agua de mar.

- Nivel de Complejidad del pretratamiento.

- La conversión de trabajo.

- Eficiencia de los sistemas de bombeo.

- Recuperación de la energía existente en la salmuera de rechazo.

- Permeabilidad y el paso de sales de las membranas.

- Las pérdidas.

- Presión necesaria en la impulsión del agua desalinizada. 


\section{Modelo de sistema de recuperación de energía con intercambiadores de presión y membranas}

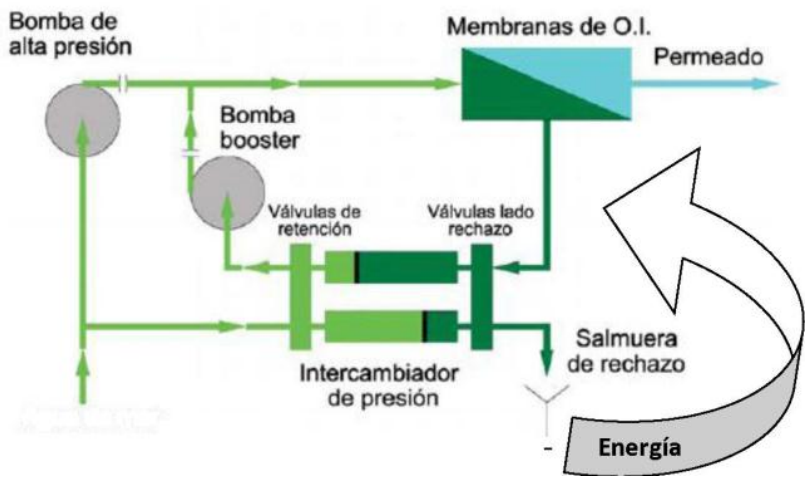

Figura 3. Esquema Desalinizador, tomado de Fariñas (2008).

Con base en investigaciones recientes y esquemas iniciales modelados computacionalmente en el grupo de investigación CONVERGÍA y DINÁMICA DE FLUIDOS, se obtuvieron los siguientes resultados:

Tabla 1. Consumo específico de energía $\left(\mathrm{Kwh} / \mathrm{m}^{3}\right)$ Salinidad $39 \%$ Conversión 45\%, Intercambiadores de presión.

\begin{tabular}{ccccc}
\hline $\begin{array}{c}\text { Grado de } \\
\text { complejidad }\end{array}$ & Proceso & $\begin{array}{c}\text { Requerido } \\
\text { por el } \\
\text { proceso }\end{array}$ & $\begin{array}{c}\text { Por la ineficiencia } \\
\text { de los bombeos }\end{array}$ & $\begin{array}{c}\text { Consumo } \\
\text { específico } \\
\text { total }\end{array}$ \\
\hline Alto & Convencional & 0,48 & 0,15 & 0,53 \\
Medio & Avanzado & 0,42 & 0,13 & 0,55 \\
& Convencional & 0,41 & 0,12 & 0,53 \\
Bajo & Avanzado & 0,35 & 0,10 & 0,45 \\
& Convencional & 0,29 & 0,07 & 0,36 \\
& Avanzado & 0,29 & 0,03 & 0,37 \\
\hline
\end{tabular}

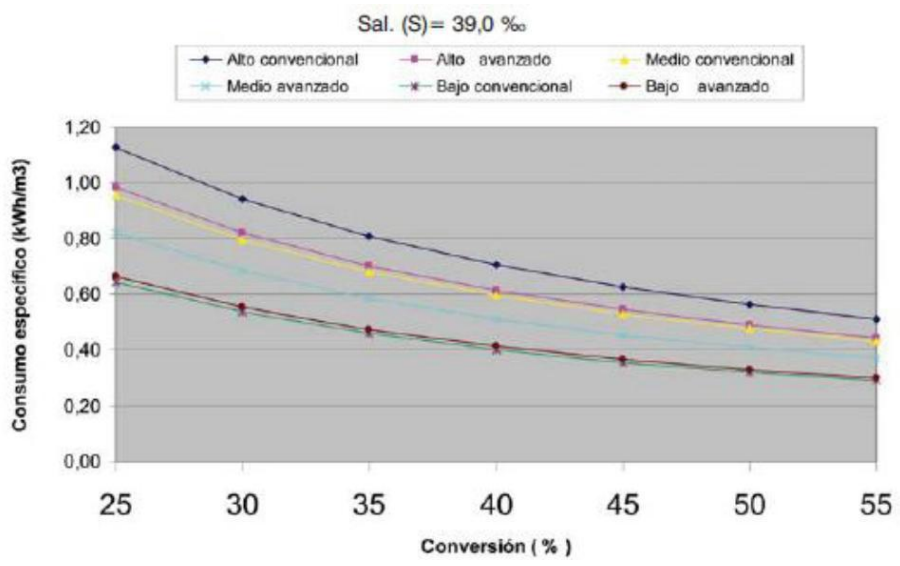

Figura 4. Evolución del consumo específico de energía en función de trabajo y del grado de complejidad.

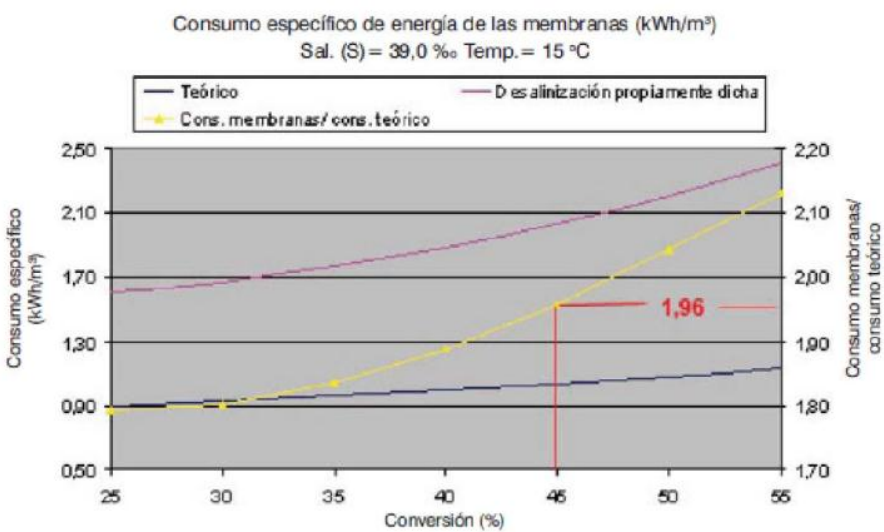

Figura 5. Relación entre el consumo real y el teórico en las membranas de ósmosis interna.

Componentes que hacen parte del modelo desalinizador son: Membranas de Osmosis Inversa Intercambiadores de Presión Sensores Bioinspirados.

\section{MEMBRANAS DE ÓSMOSIS INVERSA}

\section{¿Qué es la ósmosis?}

El fenómeno de la ósmosis está basado en la búsqueda del equilibrio. Cuando se ponen en contacto dos fluidos con diferentes concentraciones de sólidos disueltos se mezclarán hasta que la concentración sea uniforme. Si estos fluidos están separados por una membrana permeable (la cual permite el paso a su través de uno de los fluidos), el fluido que se moverá a través de la membrana será el de menor concentración de tal forma que pasa al fluido de mayor concentración. (Binnie et al. 2002).

\section{Ósmosis Inversa}

Se utiliza una presión superior a la presión osmótica, se produce el efecto contrario. Los fluidos se presionan a través de la membrana, mientras que los sólidos disueltos quedan atrás. Para desalinizar el agua es necesario llevar a cabo el proceso contrario al de la ósmosis convencional, es lo que se conoce como Ósmosis Inversa. Se trata de un proceso con membranas. Para poder forzar el paso del agua que se encuentra en la corriente de salmuera a la corriente de agua con baja concentración de sal, es necesario presurizar el agua a un valor superior al de la presión osmótica. Como consecuencia a este proceso, la salmuera se concentrará más. 


\section{INTERCAMBIADORES DE PRESIÓN}

\section{Principio básico de funcionamiento}

Los intercambiadores de presión, conocidos en inglés por las siglas WEER (Work Exchanger Energy Recovery), y que podrían trasladarse al español con las siglas SIPRE (Sistemas intercambiadores depresión para la recuperación de energía), son dispositivos que transfieren directamente la alta presión de la salmuera de rechazo al agua de mar sin convertirla previamente en energía mecánica de rotación. Imaginemos un cilindro provisto en su interior de un disco cuyo rozamiento sea mínimo.

Supongamos que un lado de dicho cilindro se conecte con el agua de mar que hay que enviar hacia las membranas y que se encuentra a una presión Pa (presión de alimentación). Supongamos igualmente que dicha conexión se realice mediante unas 8 válvulas de retención dispuestas como muestra la figura 6 y que el otro extremo se conecte mediante la válvula $\mathrm{V} 1$ con la salmuera de rechazo procedente de las membranas y que se encuentra a una presión Ps (presión de salida) y con el drenaje que se encuentra a la presión atmosférica Po a través de la válvula V2.

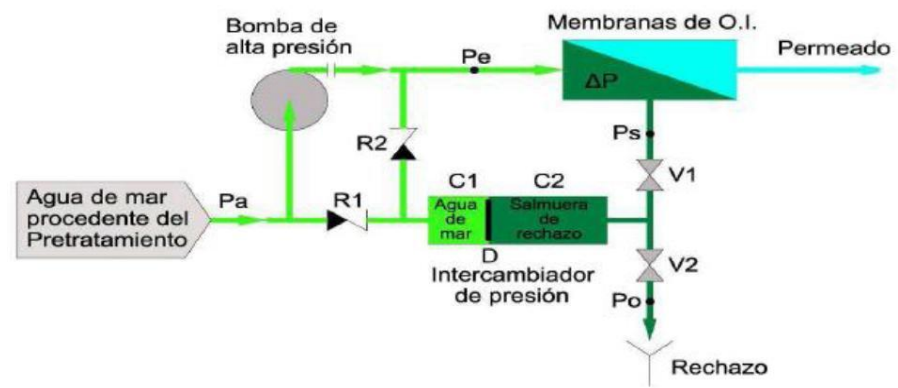

Figura 6. Esquema de la conexión de un intercambiador de presión, tomado de Fariñas (2008).

Cuando la válvula V2 está abierta y la V1 cerrada, como se recoge en la figura 7 , la salmuera de rechazo del interior del cilindro está en contacto con la atmósfera siendo desplazada por el agua de mar a baja presión que, a través de la válvula de retención R1, penetra en la cámara C1 llenándola al desplazar el disco D hacia la derecha.

Cuando el pistón llega al extremo izquierdo A, se cierra la válvula V1 abriéndose la V2 repitiéndose de nuevo el ciclo. El dispositivo descrito en la figura 5, necesita resolver dos problemas para funcionar correctamente $\mathrm{y}$ ser eficiente energéticamente. El primer problema es que la transferencia de presión al agua de mar por parte de la salmuera debe ser continua. En el caso descrito, mientras el disco D se mueve hacia la derecha, no impulsa agua de mar, lo que no es aceptable para las 10 membranas ya que se quedarían sin agua. Este problema se resuelve instalando en paralelo al menos un segundo cilindro cuyo funcionamiento debe estar desfasado respecto al primero, de manera que las membranas estén recibiendo permanentemente agua de mar a alta presión procedente del intercambiador de presión. (Ver Shymway Scott A., "The work exchanger for SWRO energy recovery", International Desalination \& Water Reuse Quarterly, February/March 1999).

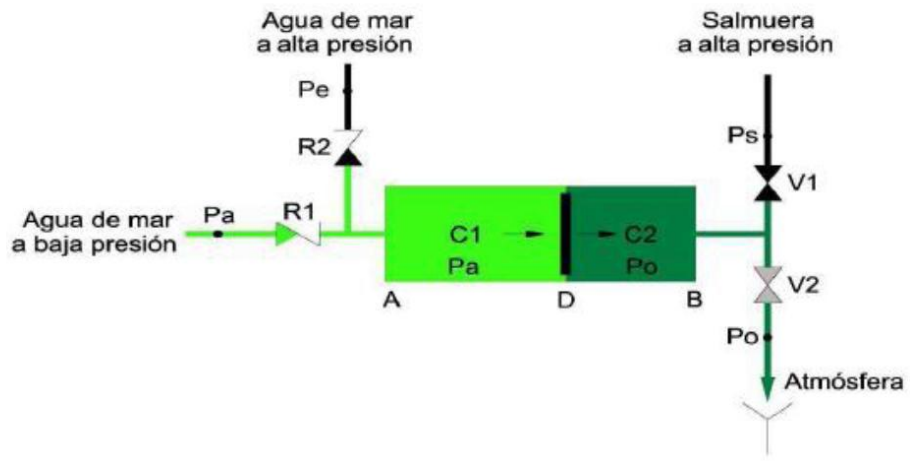

Figura 7. Alimentación con agua de mar tomado de Fariñas (2008).

El segundo problema es que la presión con la que sale la salmuera de rechazo (Ps), debido a la pérdida de carga que sufre al atravesar las membranas, es inferior a la presión con la que el agua de mar entra a éstas (Pe). Consecuentemente la presión con la que el agua de mar sale de los intercambiadores de presión es ligeramente inferior a la requerida por las membranas. Este problema se resuelve instalando una bomba booster a la salida de los intercambiadores de presión tal y como se muestra en la figura 3. La bomba booster impulsa un caudal de agua de mar ligeramente inferior al caudal de la salmuera de rechazo y la presión diferencial que aporta debe compensar la pérdida de carga que sufre la salmuera tanto en las membranas como en las tuberías y válvulas.

La bomba de alta presión debe suministrar por tanto el caudal de agua de mar que falta, es decir, un caudal ligeramente superior al de permeado como es mostrado en el modelo sugerido.

\section{Sensores Bioinspirados}

Los sensores bioinspirados son dispositivos construidos por medio de hardware configurables y sistemas electrónicos que emulan la forma de diagnosticar el procesamiento de información y resolución de problemas que hacen uso los sistemas biológicos. 
Estos sensores (que están en desarrollo y pruebas; CONVERGÍA 2010) tienen la forma de pez donde adquieren las características físico-químicas del mar y el clima, adicionalmente energéticamente aprovecha las olas para ser autónomo y envía las señales de información y control de manera acústica a radio-modem en el desalinizador definiendo la operación de éste.

Previniendo posibles amenazas naturales que han sido presentadas en la región de Tumaco (Tsunami en 1979), monitoreo de impactos ambientales como concentraciones de salinidad entre otras propiedades del agua.

Bajo una racionalidad ambiental Leff (2004) que se explica posteriormente, el ecosistema marino y costero de Tumaco es necesario implantar sensores que realicen monitoreo continuo haciendo el mínimo desequilibrio del Ecosistema.

\section{El Análisis Multicriterio}

Se configura entonces el Análisis Multicriterio como una herramienta útil al momento de la evaluación de la sustentabilidad. A continuación se describe su metodología y los estudios relacionados con el tema.

La Evaluación Multicriterio se vuelve una evaluación social cuando toma en cuenta la noción de actores de forma participativa y transparente, captando estos valores legítimos sin darles pesos a los criterios. Esto genera una presión para tomar en cuenta varias dimensiones de política (económica, ambiental, social) y darles la misma importancia a todas.

Estas dimensiones se transforman en objetivos y en criterios, por ello si se da más peso a alguna se da más importancia a uno o a otro grupo social. Entonces, otorgar pesos en una evaluación multicriterio social es "técnicamente difícil, pragmáticamente no deseable y éticamente inaceptable" (Munda 2002).

Sin embargo el atributo más apreciable de la Evaluación Multicriterio Social (EMS) es su flexibilidad frente a conceptos como la inconmensurabilidad y la multidimensionalidad. Basados en esto, para evaluar la sustentabilidad del agua dulce, la EMS maneja las distintas dimensiones de análisis que implica la inconmensurabilidad de valores, siendo flexible frente a la incertidumbre y complejidad de los temas ambientales, sociales y económicos, permitiendo hacer una evaluación basada en procesos racionales y con participación activa de los involucrados. El ejercicio entonces se basa en la elección de las alternativas de acuerdo a los criterios introducidos, respetando la legitimidad de cada criterio hasta seleccionar una alternativa que satisfaga mejor las demandas de cada sector. Metodológicamente tiene la habilidad de evaluar las alternativas aún a través de criterios que tienen distinta escala como datos cuantitativos, cuantitativos difusos o cualitativos.

Conceptualmente la EMS también es compatible con la visión teórica de la economía ecológica que, en comparación con la economía ambiental, propone la evaluación de la sustentabilidad a través de Indicadores de Sustentabilidad Fuerte. Estos indicadores buscan evaluar la sustentabilidad partiendo de la multidimensionalidad y la consideración, en el desempeño de un recurso, de aspectos como físicos, energéticos, sociales aparte de los económicos, asumiendo una comparabilidad débil de valores. Por otra parte la economía ambiental, como extensión de la economía neoclásica propone la utilización de Indicadores de Sustentabilidad Débil que reducen la problemática hacia una sola dimensión: la monetaria, es decir asumiendo una comparabilidad fuerte de valores. Esta investigación utiliza la noción conceptual de la evaluación de la sustentabilidad basada en la Economía Ecológica.

De esta forma la EMS permite evaluar la sustentabilidad del agua dulce a partir de la desalinización del agua de mar (salada y Salobre), pero tomando en cuenta los diversos y heterogéneos factores que implica su estudio, asumiendo la multidimensionalidad del proceso y generando una elección basada en un proceso lógico de toma de decisión transparente.

Se han desarrollado muchos métodos de evaluación multicriterio (Munda 2002), sin embargo debido a la versatilidad de manejo de información, a la capacidad de manejar información cualitativa, cuantitativa y difusa a la vez y por tener un módulo que permite ingresar los valores y puntos de vista de distintos grupos sociales, esta investigación utilizará el método NAIADE (Novel Approach to Imprecise Assessment and Decision Environments). El método contiene la matriz de impacto en donde se ingresan los datos tanto de criterios, los cuales pueden ser cuantitativos (determinísticos, estocásticos, difusos o numéricos y difusos) o cualitativos.

Adicionalmente cuenta con una matriz de Equidad que permite ingresar a los sectores o grupos sociales que tienen algún grado de interés en la problemática a evaluar, para luego analizar los conflictos entre los diferentes grupos de interés y la posible formación de coaliciones, a través de una evaluación lingüística de las alternativas realizadas por cada grupo. De esta forma NAIADE calcula el orden de las alternativas de acuerdo a los criterios de evaluación (por ejemplo soluciones compromiso), proporciona indicios sobre la distancia de las posiciones de los diferentes grupos de 
interés (por ejemplo posibilidades de convergencia de intereses o formación de coaliciones) y sitúa las alternativas de acuerdo a los impactos o preferencias de los actores.

\section{Parámetros en el Análisis Multicriterio Social en Tumaco}

La sustentabilidad es un tema que engloba variables de distintas características; entre otras, temporales, dinámicas, cuantitativas, cualitativas que en ciertos casos éstas no son "rendibles". Se presenta entonces un problema al tratar de reducir a una sola cifra o al uso de un solo criterio cuando evaluamos la sustentabilidad de un recurso, de allí que surja controversia hasta en la definición de sustentabilidad y desarrollo sustentable.

Tratando de conceptualizar acorde a los consensos más generalizados, aquí se utiliza la definición que hiciera en 1987 la Comisión Mundial sobre Medio Ambiente y Desarrollo de las Naciones Unidas al desarrollo sustentable como "el desarrollo que cumple con las necesidades del presente sin comprometer la habilidad de las generaciones futuras para cumplir con sus propias necesidades" (Reporte de la Comisión Mundial sobre Medio Ambiente y Desarrollo 1987).

Esto sugiere que el recurso del agua dulce será sustentable si al menos cumple con tres requisitos: que exista en la cantidad y la calidad necesaria y que además se mantengan a lo largo del tiempo. Ahora bien, en el contexto del estudio, ¿Qué significa escasez de agua dulce y a su vez este recurso se mantenga en calidad y en cantidad para el uso de generaciones actuales y futuras? ¿Bajo qué criterios se decidirá cuándo se ha llegado a esa cantidad y calidad 'necesaria'? ¿Qué categorías deberían estar incluidas en la agregación de la calidad y cantidad del agua para su suministro? Las restricciones de la sustentabilidad y la racionalidad ambiental abarcan muchos más elementos de los que pueden aparecer a simple vista por lo que es necesario desagregarlos para identificar y comprenderlos para luego someterlos a evaluación.

Las actuales concepciones de calidad y cantidad de agua necesarias subestiman la existencia de dimensiones de análisis que envuelven el estudio de su sustentabilidad. En primer lugar, el requisito de 'cantidad del agua' se ha ligado a la cantidad de agua abastecida y si ésta es deficiente en función del consumo de una ciudad. Sin embargo la disponibilidad, bajo un contexto de sustentabilidad, va mucho más allá de éste concepto, pues se debe comprender que el agua no es un stock o un resultado de un proceso productivo planificable, sino un flujo circular que obedece a un ciclo natural dependiente de la tierra y de los seres que interactúan en ella y con ella, como ecosistema energético abierto, sumergido en procesos de incertidumbre. La disponibilidad del agua depende en última instancia del equilibrio natural que exista en los ecosistemas que 'producen' agua, en función de variables que van desde la calidad de cuencas hidrográficas hasta la forma en cómo regresa a la naturaleza el agua utilizada, pasando por las variaciones climáticas de la región. Esta noción complica la medición de la disponibilidad del agua debido a que influyen factores heterogéneos que no siempre se pueden apreciar de forma cuantitativa y en ciertos casos cuando aparecen de forma cuantitativa, no hay certeza sobre la precisión de su medida." A partir de allí se pueden identificar múltiples 'servicios' que brinda el agua entre los cuales están los más populares como el uso humano para consumo, industria, agricultura y otros quizás no tan populares pero no menos importantes como los servicios ambientales, sociales e inclusive culturales.

Por otra parte está la condición de que el agua se mantenga en 'calidad necesaria'. Existe también la concepción simplista de entender la calidad como el cumplimiento de estándares necesarios para su consumo, subestimando los estándares necesarios para los otros usos que tiene el agua. En este contexto cabe preguntarse, ¿Se ha realizado una evaluación sistemática sobre los niveles de calidad que debe tener el agua de acuerdo al uso que se le va a dar?, ¿Existe al menos una sectorización que permita identificar el uso que se dará al agua para luego optimizar una asignación de calidad acorde a la demanda segmentada? La respuesta negativa a estas preguntas, no solo en el contexto local, evidencia la necesidad de comprender al requerimiento de calidad de una forma más integral y agregada. Ambas restricciones de la sustentabilidad del agua, tanto la calidad y la cantidad se vuelven aún más difíciles de operacionalizar si se toma en cuenta que la sustentabilidad condiciona a que las variables se mantengan a lo largo del tiempo. La Figura 8 resume este concepto.

Estos elementos configuran un escenario en Tumaco cuyo intento de evaluación requiere la participación de las comunidades, que permitan por medio de sus experiencias manejar múltiples realidades del problema y sobre escenarios complejos y con incertidumbre incorporando en sitio los sensores bioinspirados haciendo contínuo y dinámico la recolección de datos del ecosistema.

La Evaluación Multicriterio Social permite realizar este tipo de ejercicios debido a que compara alternativas sobre una base racional (siendo de interés una Racionalidad ambiental), sobre diferentes escalas de valores, tomando en cuenta criterios cuantitativos y cualitativos, respetando los lineamientos de multidimensionalidad, a la vez que no busca maximizar las dimensiones sino encontrar soluciones 
compromiso entre los diferentes implicados del conflicto. (Martinez-Alier et al. 2008).

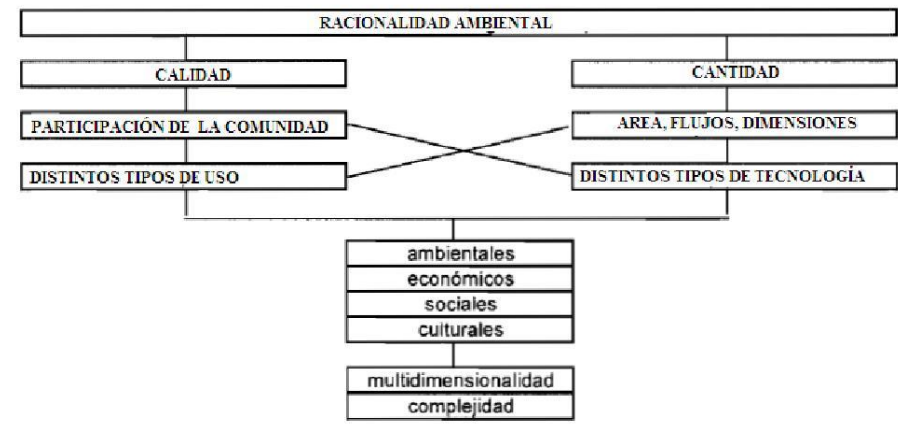

Figura 8. Diagrama de Racionalidad ambiental.

La principal ventaja del método multicriterio es que hace posible la consideración de grandes cantidades de información, relaciones entre los participantes / afectados y los objetivos de cada uno, lo cual esta generalmente presente en el mundo real. (ldem: 281). La conceptualización del Análisis Multicriterio deviene de la discusión de la sustentabilidad y su relación con las ciencias que promueven éste estudio.

\section{CONCLUSIONES}

Bajo una producción y consumo sostenible de agua dulce, el hacer uso de intercambiadores de presión para el proceso de desalinización minimiza el consumo energético en un orden del $40 \%$. El consumo específico de energía se reduce a medida que aumenta la temperatura del agua del mar.

El análisis multicriterio social permite tomar decisiones con datos cuantitativos y cualitativos siendo un referente para el diseño del sistema desalinizador híbrido con el apoyo de la comunidad. Donde los pasos a seguir según el método NADAIE (desarrollado por el Profesor Giuseppe Munda) para conformar la matriz son:

- Clasificar las alternativas de acuerdo a un conjunto de criterios de evaluación (ej. solución(es) compromiso).

- Proporcionar indicios sobre la distancia de las posiciones de los diferentes grupos de interés (ej. posibilidades de convergencia de intereses o formación de coaliciones).

- Situar las alternativas de acuerdo a los impactos o preferencias de los actores.
Este estudio es un primer paso para continuar en el desarrollo y puesta en sitio de sistemas desalinizadores aprovechando las características de la zona costera para disminuir el costo del consumo energético de la desalinización y gestión integrada del agua.

\section{REFERENCIAS}

CAMPOS, N. 2004. Contribuciones en ciencias del mar en Colombia. Investigación y desarrollo de territorios promisorios. REMAR. Universidad Nacional, Bogotá. 255 pp.

FARIÑAS, M. 2008. Aspectos energéticos de la desalinización. Ambienta: 42-48.

LEFF, E. 2004. Racionalidad Ambiental: La reapropiación de la naturaleza. Siglo XXI Editores, México D. F. 509 pp.

MALDONADO, C. 2005. Complejidad de las ciencias y ciencias de la complejidad. Universidad Externado de Colombia, Colección PRETextos27. Bogotá, 165 pp.

MARTINEZ-ALIER, J. MUNDA, G. \& O'NEILL, J.. 2008. Weak comparability of values as a foundation for ecological economic. Ecological Economics 26: 277-286.

MUNDA. G. 2002. Métodos Multicriterio Para la Evaluación Ambiental Integrada. Universidad Autónoma de Barcelona. Mimeo.

NIVIA, A. PÉREZ, C. \& SEPULVEDA, J. . 2003. Geomorfología y Geología de la plancha 383 Ingeominas, Tumaco: 39.

PAHL-WOSTL, C. KABAT, P. \& MÖLTGEN, J. 2008. Adaptive and Integrated Water Management Coping with Complexity and Uncertainty. Springer, Berlín Heidelberg. 439 pp.

URRUTIA, F. 2001. Evolución Global de la Capacidad Instaladora de Plantas Desaladoras. Noticias. AEDYR. Madrid, No. 1

ZARZA, E. 2005. Desalación del Agua Mediante Energías Renovables. Encuentro Medioambiental Almeriense: en busca de soluciones. CIEMAT- Plataforma Solar de Almería.

ZUÑIGA, O. \& PADILLA, L. 1993. Exploración Geofísica en la Costa Pacífica de Nariño (Colombia). Revista de Ciencias (8): $41-49$. 